\title{
Perfil do nutricionista do programa nacional de alimentação escolar na região Nordeste do Brasil
}

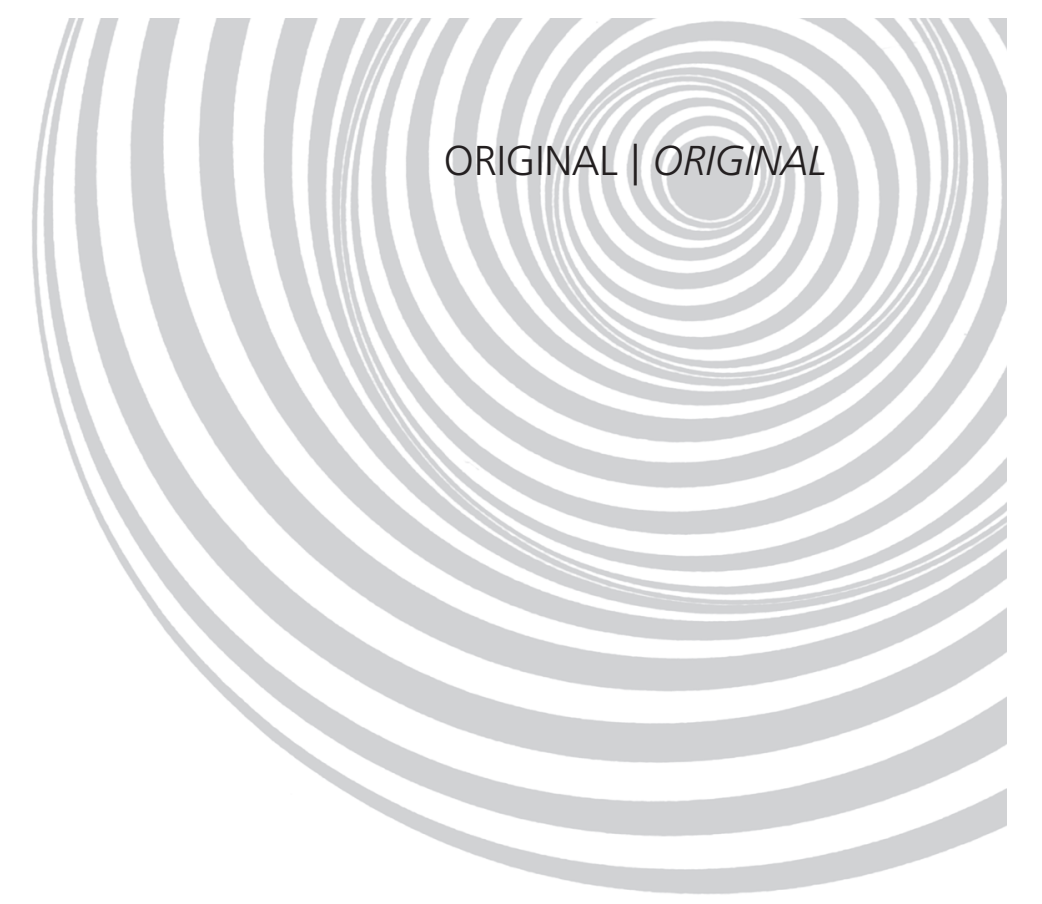

\author{
Profile of the national school food \\ program dietician in the \\ Brazilian Northeast
}

\author{
Adriana Lima MELLO' \\ Permínio Oliveira VIDAL JÚNIOR² \\ Lílian Ramos SAMPAIO' \\ Lígia Amparo da Silva SANTOS ${ }^{1}$ \\ Maria do Carmo Soares de FREITAS' \\ Gardênia Abreu Vieira FONTES ${ }^{1}$
}

RE S U M O

\section{Objetivo}

Analisar o perfil do nutricionista atuante no Programa Nacional de Alimentação Escolar, participante das formações realizadas pelo Centro Colaborador em Alimentação e Nutrição do Escolar da Universidade Federal da Bahia nos Estados do Nordeste, no ano de 2007.

\section{Métodos}

Estudo descritivo exploratório com amostragem não probabilística de 434 nutricionistas. Analisaram-se variáveis relacionadas à formação acadêmico-profissional, à atuação profissional, às atividades desenvolvidas no Programa Nacional de Alimentação Escolar e variáveis sociodemográficas. Utilizou-se o teste Qui-quadrado de Pearson, através do programa Statistical Package for the Social Sciences, para verificar associações entre o tempo de atuação no Programa Nacional de Alimentação Escolar e as atividades desenvolvidas.

\section{Resultados}

A maior parte era do sexo feminino (97,2\%), na faixa de 20 a 30 anos $(42,7 \%)$ e possuíam mais de dez anos de formado (40,2\%), predominando o vínculo empregatício efetivo por tempo parcial no Programa (53,3\%). O rendimento mensal auferido pela maior parte dos nutricionistas estava situado entre 2-5 salários-mínimos

\footnotetext{
${ }^{1}$ Universidade Federal da Bahia, Escola de Nutrição, Departamento Ciência da Nutrição. Av. Araújo Pinho, 32, Canela, 40110-150, Salvador, Bahia, Brasil. Correspondência para/Correspondence to: AL MELLO. E-mail: <amello@ufba.br>.

2 Universidade Federal do Recôncavo da Bahia, Centro de Ciências da Saúde. Santo Antônio de Jesus, BA, Brasil.
} 
120 | AL MELO et al.

$(64,1 \%)$. Dos nutricionistas que realizavam frequentemente as atividades analisadas, a maior parte possuía 2-5 anos de Programa Nacional de Alimentação Escolar $(p<0,05)$.

\section{Conclusão}

O nutricionista no âmbito do Programa Nacional de Alimentação Escolar assume responsabilidades que fazem interface com outras áreas do conhecimento, destacadamente educação e administração. Paralelamente, apesar da satisfação na atuação profissional, os participantes sentem-se pouco satisfeitos em relação às condições de trabalho.

Termos de indexação: Alimentação escolar. Nutricionista. Perfil profissional.

\section{A B S T R A C T}

\section{Objective}

This study analyzed the profile of dieticians who work for the National School Food Program and participated in training courses offered by the Schoolchildren's Food and Nutrition Collaborator Center of the Northeastern states in 2007.

\section{Methods}

This descriptive, exploratory study used nonprobability sampling to recruit 434 dieticians. Education, work experience, work, activities performed at National School Food Program and sociodemographic variables were analyzed. Pearson's chi square test was used to investigate possible associations between time working for National School Food Program and performed activities.

\section{Results}

Most participants were females (97.2\%) aged 20 to 30 years (42.7\%) and graduated more than ten years ago (40.2\%). Most were also working only part time in the program (53.3\%). The income of most dieticians ranged from 2-5 minimum salaries (64.1\%). Most dieticians who frequently performed the analyzed activities had been in National School Food Program for 2-5 years $(p<0.05)$.

\section{Conclusion}

National School Food Program dieticians take on responsibilities that overlap with other areas of knowledge, especially education and administration. In parallel, despite the professional satisfaction, the participants were not very satisfied with their work conditions.

Indexing terms: School food. Nutritionist. Professional profile.

\section{N T R O D U Ç Ã O}

O Programa Nacional de Alimentação Escolar (PNAE) tem como princípios a equanimidade, a universalidade, a equidade, a descentralização e a participação social, representando um dos mais antigos e importantes programas na área de Alimentação e Nutrição do País, atendendo atualmente, com a Lei $n^{\circ} 11.947$, de 16 de junho de 2009, cerca de 47 milhões de estudantes da educação básica e de jovens e adultos em todo Brasil ${ }^{1}$.

De acordo com a Resolução/CD/FNDE $n^{\circ}$ 038/2009, que dispõe sobre o atendimento da alimentação escolar aos alunos da educação básica no PNAE, a finalidade do Programa consiste em contribuir para o crescimento e o desenvolvimento biopsicossocial, a aprendizagem, o rendimento escolar e a formação de práticas alimentares saudáveis dos alunos, por meio de ações de educação alimentar e nutricional e da oferta de refeições que cubram as necessidades nutricionais, durante o período letivo, de pré-escolares e escolares das redes públicas, alunos de creches públicas, filantrópicas, escolas indígenas e quilombolas $^{2}$. Adicionalmente, as diretrizes da alimentação escolar preveem a aplicação de uma alimentação saudável e adequada, incluindo educação alimentar e nutricional no processo de ensino, pautada na universalidade e na segurança alimentar e nutricional' ${ }^{1}$. 
As atividades educativas em nutrição devem ser assumidas no cotidiano dos serviços de alimentação escolar, pois podem e devem sustentar as ações promotoras de saúde, particularmente no âmbito escolar, que representa um espaço privilegiado para o aprendizado permanente ${ }^{3,4}$.

Nesse sentido, o papel do nutricionista ganha destaque no PNAE, na medida em que ele pode contribuir para imprimir mudanças no hábito alimentar do escolar, na perspectiva das práticas alimentares saudáveis e na busca da Segurança Alimentar e Nutricional, embora ainda incipientes no ambiente escolar-6.

Nas duas últimas décadas, têm-se registrado avanços significativos no Programa, a exemplo das mudanças no seu aparato legal e da crescente contratação de nutricionistas, consolidando assim esse profissional como responsável técnico pelas ações da alimentação escolar. A ele é atribuída, dentre outras funções, a responsabilidade de "coordenar o diagnóstico e o monitoramento do estado nutricional dos estudantes, planejar o cardápio da alimentação escolar de acordo com a cultura alimentar, o perfil epidemiológico da população atendida e a vocação agrícola da região, acompanhando desde a aquisição dos gêneros alimentícios até a produção e distribuição da alimentação, bem como propor e realizar ações de educação alimentar e nutricional nas escolas"1.

A ele cabe também programar, elaborar e avaliar os cardápios, tendo em vista a adequação aos perfis epidemiológicos e às faixas etárias das populações atendidas, o respeito aos hábitos alimentares de cada localidade e à sua vocação agrícola, de maneira a promover hábitos alimentares saudáveis. Deve ainda ser respeitada a Resolução n 358/2005 do Conselho Federal de Nutricionistas (CFN) e suas substituições, a qual dispõe sobre as atribuições do nutricionista no âmbito do Programa de Alimentação Escolar 1,7.

Assim, conforme salientado por Santos et al. ${ }^{8}$, mudanças estruturais, bem como a concepção da alimentação como direito humano e as transições alimentar e nutricional, dentre outros fatores, determinaram o surgimento de novas áreas de atuação para o nutricionista, as quais consequentemente requerem abordagens diferenciadas desse profissional.

Entretanto, no Brasil, não há dados disponíveis sobre o perfil do nutricionista responsável técnico do PNAE. Usualmente, os estudos referem-se à avaliação do profissional em outras áreas de atuação, a exemplo da nutrição clínica e da atenção alimentar para coletividades, não se contemplando a análise quanto à sua atuação naquele Programa.

Considerando a existência de uma lacuna em relação ao conhecimento sobre a atuação do nutricionista do PNAE, estudos que possam revelar a sua prática profissional, na condição de responsável técnico do Programa, são de fundamental importância para identificar estratégias e redirecionar o processo de trabalho desse profissional, aprimorando suas competências na perspectiva da promoção da saúde dos escolares e da contribuição para o avanço do ensino no país. Para tanto, propõe-se neste trabalho analisar o perfil do nutricionista atuante no PNAE, participante das formações realizadas pelo Centro Colaborador em Alimentação e Nutrição do Escolar-UFBA em todos os estados do Nordeste durante o ano de 2007.

\section{M É T O D O S}

Trata-se de um estudo descritivo exploratório, realizado durante as formações de nutricionistas atuantes no Programa Nacional de Alimentação Escolar (PNAE), na Região Nordeste do país, no período compreendido entre março e novembro de 2007, como parte integrante das ações de capacitação do Centro Colaborador em Alimentação e Nutrição do Escolar da Universidade Federal da Bahia (CECANE-UFBA). O Centro foi criado em 2006 pelo Fundo Nacional de Desenvolvimento da Educação (FNDE) com o objetivo de fortalecer, subsidiar e otimizar as ações desse órgão e dos agentes do Programa de Alimentação e Nutrição Escolar. Além desse, sete Centros Cola- 
boradores foram criados no Brasil, com abrangência regional do plano de ações. Portanto, coube ao CECANE-UFBA o desenvolvimento de ações na Região Nordeste do Brasil no ano de 2007, sendo a sua área de abrangência posteriormente restrita aos estados da Bahia, Sergipe e Alagoas.

Dentre as metas estabelecidas para o ano de 2007, o CECANE-UFBA deveria capacitar 500 nutricionistas na Região Nordeste. A Bahia contemplou o maior número de profissionais, uma vez que é o maior estado da região, tendo sediado a formação em quatro polos (Salvador, Feira de Santana, Paulo Afonso e Itabuna). Nos demais estados, o Programa de Formação dos agentes do PNAE foi desenvolvido nas cidades de Aracaju, Maceió, Recife, João Pessoa, Natal, Fortaleza, São Luís e Teresina.

A Tabela 1 contempla, por estado, o número de nutricionistas capacitados pelo CECANEUFBA e cadastrados no FNDE, de acordo com informações prestadas por esse órgão. Entretanto, os dados cadastrais referem-se até o mês de julho de 2007. Foi realizada uma amostragem não probabilística, por conveniência, cujo tamanho e seleção não foram planejados. Dessa maneira, os profissionais que estavam efetivamente em exercício no PNAE foram identificados a partir de mecanismos que incluíram, entre outros, o contato com a União Nacional dos Dirigentes Municipais de Educação (UNDIME), com as secretarias de educação estaduais e municipais, com os Conselhos Regionais de Nutricionistas (CRN), bem como com gestores escolares estaduais e municipais e coordenadores da alimentação escolar nos municípios. A busca ativa mencionada e a inscrição dos profissionais foram realizadas pela equipe executora contratada pelo CECANE-UFBA.

Os 519 nutricionistas capacitados receberam o questionário juntamente com o termo de consentimento explicitando os objetivos do estudo. Dos participantes, 434 devolveram o questionário devidamente preenchido com as informações solicitadas, integrando a amostra e correspondendo a uma taxa de adesão de 83,6\%.
Tabela 1. Número de nutricionistas cadastradas no FNDE e participantes do Programa de Formação do CECANE-UFBA. Salvador (BA), 2007.

\begin{tabular}{lccccc}
\hline Estado & $\begin{array}{c}\text { Nutricionistas } \\
\text { cadastradas } \\
\text { no FNDE }\end{array}$ & & \multicolumn{2}{c}{$\begin{array}{c}\text { Nutricionistas } \\
\text { participantes da } \\
\text { formação }\end{array}$} \\
\cline { 2 - 3 } \cline { 5 - 6 } Bahia & $\mathrm{n}$ & & $\mathrm{n}$ & $\%$ \\
Alagoas & 241 & & 126 & 52,28 \\
Paraíba & 73 & & 37 & 50,68 \\
Pernambuco & 134 & & 62 & 46,27 \\
Rio Grande do Norte & 132 & & 51 & 38,63 \\
Ceará & 110 & & 70 & 63,63 \\
Maranhão & 136 & & 84 & 61,76 \\
Piauí & 130 & & 33 & 25,38 \\
Total & 143 & & 56 & 39,16 \\
\hline
\end{tabular}

FNDE: Fundo Nacional de Desenvolvimento da Educação; CECANEUFBA: Centro Colaborador em Alimentação e Nutrição do Escolar Universidade Federal da Bahia.

O instrumento de coleta de dados foi elaborado com base nas atribuições do nutricionista no âmbito do PNAE, conforme propõem as Resoluções FNDE n 32/06 e CFN n 358/057', vigentes na época, com o intuito de conhecer as condições de trabalho e a atuação do nutricionista na Região Nordeste. O formulário autoaplicável incluiu quatro blocos de perguntas, sendo o primeiro referente aos dados pessoais, enquanto os demais contemplaram aspectos relacionados à formação acadêmico-profissional, atuação profissional e atividades desenvolvidas no PNAE. Foram analisadas variáveis sociodemográficas (sexo, idade, renda categorizada em salário-mínimo) e variáveis relacionadas à formação profissional, às atividades técnicas desenvolvidas e ao grau de satisfação em relação às condições de trabalho e à sua atuação profissional. O tempo de atuação no PNAE foi categorizado em anos, considerando os seguintes pontos de corte: $<1,1-2,2-5$ e $>10$.

As informações coletadas foram tabuladas e processadas em banco de dados, utilizando-se o Statistical Package for the Social Sciences, versão 13.0 (SPSS), sendo analisadas frequências das variáveis que integraram a base de dados. Foi realizado o teste Qui-quadrado de Pearson para 
verificar associação entre as atividades do nutricionista no PNAE e o tempo de atuação no Programa, utilizando nível de significância de 0,05.

A pesquisa foi aprovada pelo Comitê de Ética em Pesquisa da Escola de Nutrição da UFBA, sob Parecer no 111/2007, de 08/08/2007.

\section{RES ULTADOS}

A distribuição percentual dos nutricionistas de acordo com as características sociodemográ- ficas, de formação, tempo de exercício profissional e vínculo com o Programa está apresentada na Tabela 2. Constatou-se que a maior parte do grupo investigado era do sexo feminino, concluiu algum tipo de pós-graduação e situava-se na faixa etária de 20 a 30 anos (42,7\%), enquanto a menor parcela estava acima dos 50 anos de idade $(6,7 \%)$. No que se refere à formação, o grupo caracterizou-se por apresentar mais de dez anos de formado, embora a parcela de recém-formados ( $<5$ anos) se destacasse logo em seguida, com $35 \%$.

Tabela 2. Distribuição dos nutricionistas atuantes no PNAE (Região Nordeste), de acordo com as características sociodemográficas, de formação, tempo de atuação e vínculo com o Programa (n=434). Salvador (BA), 2007.

\begin{tabular}{|c|c|c|c|c|}
\hline \multirow{2}{*}{ Variáveis } & \multicolumn{4}{|c|}{ Nutricionista } \\
\hline & \multicolumn{2}{|c|}{$\mathrm{n}$} & \multicolumn{2}{|c|}{$\%$} \\
\hline \multicolumn{5}{|l|}{ Sexo } \\
\hline Feminino & \multicolumn{2}{|c|}{422} & \multicolumn{2}{|c|}{97,2} \\
\hline \multicolumn{5}{|l|}{ Idade (anos) } \\
\hline $20-30$ & \multicolumn{2}{|c|}{185} & \multicolumn{2}{|c|}{42,7} \\
\hline $30-40$ & \multicolumn{2}{|c|}{140} & \multicolumn{2}{|c|}{32,3} \\
\hline $40-50$ & \multicolumn{2}{|c|}{81} & \multicolumn{2}{|c|}{18,7} \\
\hline$>50$ & \multicolumn{2}{|c|}{27} & \multicolumn{2}{|c|}{6,3} \\
\hline \multicolumn{5}{|l|}{ Anos de formado (a) } \\
\hline$<5$ & \multicolumn{2}{|c|}{151} & \multicolumn{2}{|c|}{35,0} \\
\hline $5-10$ & \multicolumn{2}{|c|}{107} & \multicolumn{2}{|c|}{24,8} \\
\hline$>10$ & \multicolumn{2}{|c|}{174} & \multicolumn{2}{|c|}{40,2} \\
\hline \multicolumn{5}{|l|}{ Possui pós-graduação } \\
\hline Sim & \multicolumn{2}{|c|}{226} & \multicolumn{2}{|c|}{52,2} \\
\hline Não & \multicolumn{2}{|c|}{207} & \multicolumn{2}{|c|}{47,8} \\
\hline \multicolumn{5}{|l|}{ Atuação no PNAE (anos) } \\
\hline$<1$ & \multicolumn{2}{|c|}{131} & & \\
\hline $1-2$ & & & & \\
\hline $2-5$ & & & & \\
\hline$>5$ & & & & \\
\hline Vínculo com o Program & & & & \\
\hline Consultoria & & & & \\
\hline Contrato & & & & \\
\hline Efetivo, tempo integral & & & & \\
\hline Efetivo, tempo parcial & & & & \\
\hline Faixa calarial & & & & \\
\hline & $n$ & $\%$ & $\mathrm{n}$ & $\%$ \\
\hline <2 salário-mínimo & 98 & 22,6 & 18 & 4,1 \\
\hline 2-5 salário-mínimo & 278 & 64,1 & 225 & 51,8 \\
\hline 5-10 salário-mínimo & 36 & 8,3 & 149 & 34,3 \\
\hline >10 salário-mínimo & 1 & 0,2 & 13 & 3,0 \\
\hline Sem informação & 21 & 4,8 & 29 & 6,7 \\
\hline
\end{tabular}

PNAE: Programa Nacional de Alimentação Escolar. 
No que diz respeito à atuação no Programa (Tabela 2), quase um terço dos nutricionistas, considerados nesta abordagem, trabalhava no PNAE há menos de um ano $(30,2 \%)$ ou entre dois e cinco anos (29,5\%). Grande parte desses profissionais não trabalhava exclusivamente na alimentação escolar, visto que $70,5 \%$ relataram o exercício profissional em outras áreas, estando entre as mais prevalentes a nutrição clínica e a alimentação coletiva.

Considerando a distribuição dos nutricionistas de acordo com o tipo de vínculo empregatício no Programa, observou-se que o vínculo efetivo por tempo parcial predominou, muito embora os vínculos temporários (consultoria e contrato) tenham somado 35\% (Tabela 2). Adicionalmente, 33,3\% informaram atender em mais de um município, com a maior parte destes exercendo a profissão em duas localidades. É ainda relevante destacar que dois profissionais disseram trabalhar em quinze municípios (dados não apresentados).

Em relação aos rendimentos dos nutricionistas participantes da pesquisa (Tabela 2), observou-se que a maioria auferia vencimentos entre 2 e 5 salários-mínimos, tanto para a renda no PNAE quanto para a renda total. Deve ser considerado ainda o percentual expressivo de nutricionistas que atingiu rendimentos inferiores a dois salários-mínimos como responsáveis técnicos do Programa.

Como informação adicional aos dados apresentados, a maioria dos nutricionistas declarou não integrar os Conselhos de Alimentação Escolar (CAE) $(92,6 \%$ ), embora $46,4 \%$ participassem com frequência de suas reuniões.

A Tabela 3 apresenta a distribuição dos nutricionistas de acordo com as atividades desempenhadas no Programa, bem como a associação destas com o tempo de atuação no PNAE. Entre as principais atividades executadas pelos nutricionistas estão: programar e elaborar cardápios; participar do processo de aquisição de gêneros alimentícios; supervisionar a produção e fornecimento das refeições; capacitar merendeiras. Por outro lado, atividades que incluem o acompanhamento da situação nutricional dos beneficiários, participação em equipes multidisciplinares, desenvolvimento de ações educativas com escolares, professores e familiares, não foram efetivadas ou foram realizadas eventualmente.

Os resultados obtidos a partir do teste Qui-quadrado de Pearson (Tabela 3), sugerem que o tempo de atuação do nutricionista no PNAE pode ter influenciado a realização das atividades, uma vez que houve associação estatisticamente significante para todas as atividades listadas. O percentual de nutricionistas que realizou as atividades com frequência foi superior no grupo que tinha entre dois e cinco anos de atuação no Programa.

As atividades relacionadas com a elaboração dos cardápios da alimentação escolar associaram-se também com o tempo de exercício profissional no PNAE, conforme apresentado na Tabela 4. Essas atividades corresponderam ao cálculo dos parâmetros nutricionais; ao planejamento, orientação e supervisão de etapas ligadas ao alimento; à realização de teste de aceitabilidade junto à clientela; ao atendimento específico para os escolares portadores de patologias e deficiências associadas à nutrição e, por fim, à elaboração de fichas técnicas das preparações que compõem o cardápio.

Observou-se que as cinco atividades que deveriam subsidiar a elaboração dos cardápios da alimentação escolar não eram realizadas integralmente. Atividades como a aplicação do teste de aceitabilidade e atendimento individualizado aos escolares portadores de patologias e deficiências vinculadas à nutrição, na grande parte dos casos, não eram realizadas ou o eram raramente. Por outro lado, outras ações eram desempenhadas frequentemente na elaboração dos cardápios, tais como: cálculo dos parâmetros nutricionais e planejamento, orientação e supervisão da seleção, compra, armazenamento, produção e distribuição dos alimentos.

O tempo de atuação do nutricionista no PNAE pode ter influenciado na realização das atividades vinculadas ao cardápio, visto que houve associação com significância estatística para as cinco atividades, de acordo com o teste Qui- 
Tabela 3. Associação entre realização de atividades no PNAE e tempo de atuação do nutricionista no Programa ( $n=434)$. Salvador (BA), 2007

\begin{tabular}{|c|c|c|c|c|c|c|c|c|c|c|c|c|c|c|c|c|}
\hline \multirow{4}{*}{$\begin{array}{l}\cdot \frac{0}{U} \\
\frac{C}{d} \\
\frac{U}{O} \\
\frac{d}{4} \\
\frac{1}{L}\end{array}$} & \multicolumn{8}{|c|}{ Assessora o CAE quanto à execução do PNAE* } & \multicolumn{8}{|c|}{ Participa do processo de aquisição de gêneros alimentícios* } \\
\hline & \multicolumn{8}{|c|}{ Tempo de PNAE (anos) } & \multicolumn{8}{|c|}{ Tempo de PNAE (anos) } \\
\hline & \multicolumn{2}{|c|}{$<1$} & \multicolumn{2}{|c|}{$1-2$} & \multicolumn{2}{|c|}{$2-5$} & \multicolumn{2}{|c|}{$>10$} & \multicolumn{2}{|c|}{$<1$} & \multicolumn{2}{|c|}{$1-2$} & \multicolumn{2}{|c|}{$2-5$} & \multicolumn{2}{|c|}{$>10$} \\
\hline & $n$ & $\%$ & $n$ & $\%$ & $n$ & $\%$ & $n$ & $\%$ & $n$ & $\%$ & $n$ & $\%$ & $n$ & $\%$ & $n$ & $\%$ \\
\hline NR & 56 & 48,3 & 32 & 27,6 & 23 & 19,8 & 5 & 4,3 & 26 & 39,4 & 13 & 19,7 & 18 & 27,3 & 9 & 13,6 \\
\hline $\mathrm{RR}$ & 36 & 26,1 & 40 & 29,0 & 42 & 30,4 & 20 & 14,5 & 21 & 30,9 & 22 & 32,4 & 21 & 30,9 & 4 & 5,9 \\
\hline RF & 29 & 17,5 & 30 & 18,1 & 62 & 37,3 & 45 & 27,1 & 73 & 25,4 & 68 & 23,7 & 88 & 30,7 & 58 & 20,2 \\
\hline SI & 10 & 71,4 & 2 & 14,3 & 1 & 7,1 & 1 & 7,1 & 11 & 84,6 & 1 & 7,7 & 1 & 7,7 & 0 & 0 \\
\hline Total & 131 & 30,2 & 104 & 24,0 & 128 & 29,5 & 71 & 16,4 & 131 & 30,2 & 104 & 24,0 & 128 & 29,5 & 71 & 16,4 \\
\hline \multirow{4}{*}{ 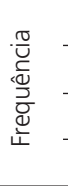 } & \multicolumn{8}{|c|}{ Programa e elabora os cardápios do PNAE* } & \multicolumn{8}{|c|}{ Supervisiona a produção e fornecimento das refeições nas escolas } \\
\hline & \multicolumn{8}{|c|}{ Tempo de PNAE (anos) } & \multicolumn{8}{|c|}{ Tempo de PNAE (anos) } \\
\hline & \multicolumn{2}{|c|}{$<1$} & \multicolumn{2}{|c|}{$1-2$} & \multicolumn{2}{|c|}{$2-5$} & \multicolumn{2}{|c|}{$>10$} & \multicolumn{2}{|c|}{$<1$} & \multicolumn{2}{|c|}{$1-2$} & \multicolumn{2}{|c|}{$2-5$} & \multicolumn{2}{|c|}{$>10$} \\
\hline & $n$ & $\%$ & $n$ & $\%$ & $n$ & $\%$ & $n$ & $\%$ & $\mathrm{n}$ & $\%$ & $n$ & $\%$ & $n$ & $\%$ & $n$ & $\%$ \\
\hline NR & 3 & 50,0 & 1 & 16,7 & 1 & 16,7 & 1 & 16,7 & 3 & 27,3 & 2 & 18,2 & 5 & 45,5 & 1 & 9,1 \\
\hline $\mathrm{RR}$ & 6 & 42,9 & 4 & 28,6 & 3 & 21,4 & 1 & 7,1 & 48 & 28,6 & 49 & 29,2 & 46 & 27,4 & 25 & 14,9 \\
\hline RF & 113 & 28,0 & 98 & 24,3 & 124 & 30,7 & 69 & 17,1 & 71 & 29,0 & 52 & 21,2 & 77 & 31,4 & 45 & 18,4 \\
\hline SI & 9 & 90,0 & 1 & 10,0 & 0 & 0 & 0 & 0 & 9 & 90,0 & 1 & 10,0 & 0 & 0 & 0 & 0 \\
\hline Total & 131 & 30,2 & 104 & 24,0 & 128 & 29,5 & 71 & 16,4 & 131 & 30,2 & 104 & 24,0 & 128 & 29,5 & 71 & 16,4 \\
\hline \multirow{4}{*}{  } & & & bora & plano & ual de & rabalh & & & Realiz & rein & nto de & nere & ras & mais & nipu & ores $^{*}$ \\
\hline & & & & o de & $\mathrm{EE}(\mathrm{ar}$ & & & & & & & $\overline{O d}$ & $\mathrm{AE}(\mathrm{ar}$ & & & \\
\hline & & & & & & & & & & & & & & & & \\
\hline & $n$ & $\%$ & $n$ & $\%$ & $n$ & $\%$ & $n$ & $\%$ & $n$ & $\%$ & $n$ & $\%$ & $n$ & $\%$ & $n$ & $\%$ \\
\hline NR & 59 & 35,1 & 36 & 21,4 & 51 & 30,4 & 22 & 13,1 & 13 & 61,9 & 5 & 23,8 & 3 & 14,3 & 0 & 0 \\
\hline $\mathrm{RR}$ & 17 & 25,8 & 17 & 25,8 & 23 & 34,8 & 9 & 13,6 & 36 & 28,1 & 34 & 26,6 & 42 & 32,8 & 16 & 12,5 \\
\hline RF & 40 & 22,1 & 49 & 27,1 & 52 & 28,7 & 40 & 22,1 & 71 & 26,4 & 64 & 23,8 & 81 & 30,1 & 53 & 19,7 \\
\hline SI & 15 & 78,9 & 2 & 10,5 & 2 & 10,5 & 0 & 0 & 11 & 73,3 & 1 & 6,7 & 1 & 6,7 & 2 & 13,3 \\
\hline Total & 131 & 30,2 & 104 & 24,0 & 128 & 29,5 & 71 & 16,4 & 131 & 30,3 & 104 & 24,0 & 127 & 29,3 & 71 & 16,4 \\
\hline
\end{tabular}

\begin{tabular}{|c|c|c|c|c|c|c|c|c|c|c|c|c|c|c|c|c|}
\hline \multirow{4}{*}{ 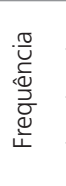 } & \multicolumn{8}{|c|}{ Monitoramento da situação nutricional dos escolares* } & \multicolumn{8}{|c|}{ Participa de equipes multidisciplinares* } \\
\hline & \multicolumn{8}{|c|}{ Tempo de PNAE (anos) } & \multicolumn{8}{|c|}{ Tempo de PNAE (anos) } \\
\hline & \multicolumn{2}{|c|}{$<1$} & \multicolumn{2}{|c|}{$1-2$} & \multicolumn{2}{|c|}{$2-5$} & \multicolumn{2}{|c|}{$>10$} & \multicolumn{2}{|c|}{$<1$} & \multicolumn{2}{|c|}{$1-2$} & \multicolumn{2}{|c|}{$2-5$} & \multicolumn{2}{|c|}{$>10$} \\
\hline & $\mathrm{n}$ & $\%$ & $\mathrm{n}$ & $\%$ & $\mathrm{n}$ & $\%$ & $\mathrm{n}$ & $\%$ & $\mathrm{n}$ & $\%$ & $\mathrm{n}$ & $\%$ & $\mathrm{n}$ & $\%$ & $n$ & $\%$ \\
\hline NR & 58 & 31,4 & 46 & 24,9 & 50 & 27,0 & 31 & 16,8 & 65 & 33,7 & 45 & 23,3 & 58 & 30,1 & 25 & 13,0 \\
\hline $\mathrm{RR}$ & 44 & 27,3 & 38 & 23,6 & 51 & 31,7 & 28 & 17,4 & 41 & 27,9 & 36 & 24,5 & 45 & 30,6 & 25 & 17,0 \\
\hline RF & 18 & 24,3 & 18 & 24,3 & 26 & 35,1 & 12 & 16,2 & 14 & 17,7 & 20 & 25,3 & 24 & 30,4 & 21 & 26,6 \\
\hline SI & 11 & 78,6 & 2 & 14,3 & 1 & 7,1 & 0 & 0 & 11 & 73,3 & 3 & 20,0 & 1 & 6,7 & 0 & 0 \\
\hline Total & 131 & 30,2 & 104 & 24,0 & 128 & 29,5 & 71 & 16,4 & 131 & 30,2 & 104 & 24,0 & 128 & 29,5 & 71 & 16,4 \\
\hline
\end{tabular}

\begin{tabular}{|c|c|c|c|c|c|c|c|c|c|c|c|c|c|c|c|c|}
\hline \multirow{3}{*}{ 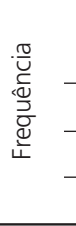 } & \multicolumn{8}{|c|}{ Tempo de PNAE (anos) } & \multicolumn{8}{|c|}{ Tempo de PNAE (anos) } \\
\hline & \multicolumn{2}{|c|}{$<1$} & \multicolumn{2}{|c|}{$1-2$} & \multicolumn{2}{|c|}{$2-5$} & \multicolumn{2}{|c|}{$>10$} & \multicolumn{2}{|c|}{$<1$} & \multicolumn{2}{|c|}{$1-2$} & \multicolumn{2}{|c|}{$2-5$} & \multicolumn{2}{|c|}{$>10$} \\
\hline & $n$ & $\%$ & $n$ & $\%$ & $n$ & $\%$ & $n$ & $\%$ & $n$ & $\%$ & $n$ & $\%$ & $n$ & $\%$ & $n$ & $\%$ \\
\hline $\mathrm{RR}$ & 59 & 28,6 & 52 & 25,2 & 62 & 30,1 & 33 & 16,0 & 58 & 28,4 & 46 & 22,5 & 62 & 30,4 & 38 & 18,6 \\
\hline RF & 34 & 23,6 & 32 & 22,2 & 51 & 35,4 & 27 & 18,8 & 8 & 14,3 & 15 & 26,8 & 22 & 39,3 & 11 & 19,6 \\
\hline SI & 10 & 83,3 & 2 & 16,7 & 0 & 0 & 0 & 0 & 11 & 64,7 & 3 & 17,6 & 3 & 17,6 & 0 & 0 \\
\hline Total & 131 & 30,2 & 104 & 24,0 & 128 & 29,5 & 71 & 16,4 & 131 & 30,2 & 104 & 24,0 & 128 & 29,5 & 71 & 16,4 \\
\hline
\end{tabular}

p<0,01. NR: Não Realiza; RR: Realiza Raramente; RF: Realiza Frequentemente; SI: Sem Informação; CAE: Conselho de Alimentação Escolar; PNAE: Programa Nacional de Alimentação Escolar. 
Tabela 4. Associação entre tempo de atuação no PNAE e atividades desenvolvidas pelo nutricionista na elaboração dos cardápios do Programa. Salvador (BA), 2007.

\begin{tabular}{|c|c|c|c|c|c|c|c|c|c|c|c|}
\hline \multirow{3}{*}{ Variáveis } & \multicolumn{8}{|c|}{ Tempo de PNAE } & & & \multirow{3}{*}{ p-valor } \\
\hline & \multicolumn{2}{|c|}{$<1$} & \multicolumn{2}{|c|}{1 a 2} & \multicolumn{2}{|c|}{2 a 5} & \multicolumn{2}{|c|}{$>5$} & \multicolumn{2}{|c|}{ Total } & \\
\hline & $n$ & $\%$ & $\mathrm{n}$ & $\%$ & $\mathrm{n}$ & $\%$ & $\mathrm{n}$ & $\%$ & $n$ & $\%$ & \\
\hline \multicolumn{12}{|c|}{ Calcula os parâmetros nutricionais com base nas recomendações nutricionais, avaliação nutricional e necessidades específicas } \\
\hline NR & 21 & $(26,9)$ & 29 & $(37,2)$ & 17 & $(21,8)$ & 11 & $(14,1)$ & 78 & $(100,0)$ & \\
\hline $\mathrm{RR}$ & 31 & $(27,7)$ & 28 & $(25,0)$ & 34 & $(30,4)$ & 19 & $(17,0)$ & 112 & $(100,0)$ & \\
\hline RF & 66 & $(29,2)$ & 45 & $(19,9)$ & 75 & $(33,2)$ & 40 & $(17,7)$ & 226 & $(100,0)$ & $<0,01$ \\
\hline SI & 13 & $(72,2)$ & 2 & $(11,1)$ & 2 & $(11,1)$ & 1 & $(5,6)$ & 18 & $(100,0)$ & \\
\hline Total & 131 & $(30,2)$ & 104 & $(24,0)$ & 128 & $(29,5)$ & 71 & $(16,4)$ & 434 & $(100,0)$ & \\
\hline \multicolumn{12}{|c|}{ Planeja, orienta e supervisiona as atividades de seleção, compra, armazenamento, produção e distribuição dos alimentos } \\
\hline NR & 11 & $(55,0)$ & 3 & $(15,0)$ & 4 & $(20,0)$ & 2 & $(10,0)$ & 20 & $(100,0)$ & \\
\hline $\mathrm{RR}$ & 26 & $(36,6)$ & 23 & $(32,4)$ & 16 & $(22,5)$ & 6 & $(8,5)$ & 71 & $(100,0)$ & \\
\hline RF & 81 & $(24,8)$ & 76 & $(23,2)$ & 107 & $(32,7)$ & 63 & $(19,3)$ & 327 & $(100,0)$ & $<0,01$ \\
\hline SI & 13 & $(81,3)$ & 2 & $(12,5)$ & 1 & $(6,3)$ & 0 & 0 & 16 & $(100,0)$ & \\
\hline Total & 131 & $(30,2)$ & 104 & $(24,0)$ & 128 & $(29,5)$ & 71 & $(16,4)$ & 434 & $(100,0)$ & \\
\hline \multicolumn{12}{|c|}{ Realiza testes de aceitabilidade do cardápio } \\
\hline NR & 35 & $(32,4)$ & 35 & $(32,4)$ & 22 & $(20,4)$ & 16 & $(14,8)$ & 108 & $(100,0)$ & \\
\hline $\mathrm{RR}$ & 49 & $(29,0)$ & 34 & $(20,1)$ & 55 & $(32,5)$ & 31 & $(18,3)$ & 169 & $(100,0)$ & \\
\hline RF & 34 & $(24,1)$ & 32 & $(22,7)$ & 51 & $(36,2)$ & 24 & $(17,0)$ & 141 & $(100,0)$ & $<0,01$ \\
\hline SI & 13 & $(81,3)$ & 3 & $(18,8)$ & 0 & 0 & 0 & 0 & 16 & $(100,0)$ & \\
\hline Total & 131 & $(30,2)$ & 104 & $(24,0)$ & 128 & $(29,5)$ & 71 & $(16,4)$ & 434 & $(100,0)$ & \\
\hline \multicolumn{12}{|c|}{ Realiza atendimento específico para os escolares portadores de patologias e deficiências associadas à nutrição } \\
\hline$N R$ & 56 & $(29,3)$ & 51 & $(26,7)$ & 52 & $(27,2)$ & 32 & $(16,8)$ & 191 & $(100,0)$ & \\
\hline $\mathrm{RR}$ & 35 & $(27,6)$ & 31 & $(24,4)$ & 41 & $(32,3)$ & 20 & $(15,7)$ & 127 & $(100,0)$ & \\
\hline RF & 26 & $(27,1)$ & 18 & $(18,8)$ & 33 & $(34,4)$ & 19 & $(19,8)$ & 96 & $(100,0)$ & 0,01 \\
\hline $\mathrm{SI}$ & 14 & $(70,0)$ & 4 & $(20,0)$ & 2 & $(10,0)$ & 0 & 0 & 20 & $(100,0)$ & \\
\hline Total & 131 & $(30,2)$ & 104 & $(24,0)$ & 128 & $(29,5)$ & 71 & $(16,4)$ & 434 & $(100,0)$ & \\
\hline \multicolumn{12}{|c|}{ Elabora fichas técnicas das preparações que compõem o cardápio } \\
\hline NR & 36 & $(34,0)$ & 28 & $(26,4)$ & 24 & $(22,6)$ & 18 & $(17,0)$ & 106 & $(100,0)$ & \\
\hline $\mathrm{RR}$ & 29 & $(27,1)$ & 24 & $(22,4)$ & 35 & $(32,7)$ & 19 & $(17,8)$ & 107 & $(100,0)$ & \\
\hline RF & 52 & $(25,9)$ & 48 & $(23,9)$ & 67 & $(33,3)$ & 34 & $(16,9)$ & 201 & $(100,0)$ & $<0,01$ \\
\hline SI & 14 & $(70,0)$ & 4 & $(20,0)$ & 2 & $(10,0)$ & 0 & 0 & 20 & $(100,0)$ & \\
\hline Total & 131 & $(30,2)$ & 104 & $(24,0)$ & 128 & $(29,5)$ & 71 & $(16,4)$ & 434 & $(100,0)$ & \\
\hline
\end{tabular}

NR: Não Realiza; RR: Realiza Raramente; RF: Realiza Frequentemente; SI: Sem Informação.

-Quadrado de Pearson (Tabela 4). O número de nutricionistas que realizavam as atividades com frequência foi maior para os que atuavam de dois a cinco anos no Programa. De modo contrário, o grupo com atuação no PNAE superior a cinco anos apresentou frequência inferior, quando comparado aos demais grupos.

A Figura 1 apresenta o grau de satisfação do nutricionista com as condições de trabalho e com sua atuação profissional. Nota-se que esse grupo de profissionais pode ser caracterizado pelo descontentamento com as condições de trabalho no Programa, uma vez que $49,1 \%$ e $14,5 \%$ revelaram estar pouco satisfeitos e insatisfeitos, respectivamente. Por outro lado, a satisfação com as condições de trabalho e com a atuação profissional foi referida por $31,8 \%$ e $46,3 \%$ dos nutricionistas, respectivamente. 


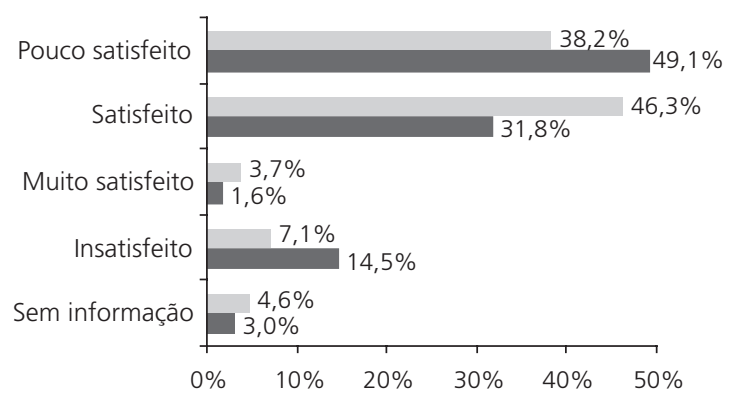

$\square$ Condições de trabalho $\square$ Atuação profissional

Figura 1. Distribuição dos nutricionistas de acordo com o nível de satisfação profissional e condições de trabalho $(N=434)$. Salvador (BA), 2007.

\section{DISCUSS Ã O}

Este estudo representa o primeiro trabalho que visa caracterizar o perfil do nutricionista atuante no Programa de Alimentação e Nutrição do Escolar no Brasil. É importante destacar que a ausência de estudos sobre o tema representou limitação significativa para exercer análise comparativa dos resultados obtidos. Entretanto, apesar dessa dificuldade, buscou-se estabelecer comparações com estudos que analisaram o perfil de nutricionistas atuantes em outras áreas da Nutrição.

Cabe ainda destacar que, do ponto de vista dos limites metodológicos, esta pesquisa não representa um estudo probabilístico e, por não se dispor de dados atualizados sobre o universo de nutricionistas cadastrados no PNAE, não se pode analisar a amostra como sendo representativa da Região Nordeste do Brasil. Os recursos disponíveis para identificação dos respondentes resultaram em uma amostra não randomizada, mas que representou $42 \%$ do total de nutricionistas da Região Nordeste cadastrados no FNDE. Além disso, o instrumento utilizado não permitiu analisar as reais condições de trabalho do nutricionista, nem tampouco o hiato existente entre o quadro de pessoal e a realização plena das atividades previstas na legislação que dispõe sobre as atribuições do nutricionista no Programa ${ }^{7}$.
O universo dos nutricionistas que participaram dos programas de formação no ano de 2007 foi composto majoritariamente pelo sexo feminino $(97,2 \%)$, com faixa etária mais jovem, entre 20 e 40 anos (75,0\%). Esses dados revelam semelhança com os encontrados no estudo realizado pelo CFN sobre a inserção profissional dos nutricionistas no Brasil em 2005, caracterizados na sua grande maioria por mulheres $(96,5 \%)$ e concentrados nas faixas etárias de 20 a 40 anos $(79,4 \%)^{10}$. Com objetivo de traçar o perfil profissional e demográfico do nutricionista brasileiro, Akutsu ${ }^{11}$ encontrou média de idade de 34,8 anos, com $42,7 \%$ dos nutricionistas situados na faixa etária de 20 a 30 anos. A predominância de recém-formados na faixa etária de 25 a 29 anos foi também verificada por Rodrigues et al. ${ }^{12}$, ao descreverem as condições de trabalho de nutricionistas egressos da Universidade Federal de Ouro Preto $(\mathrm{MG})$, em suas diferentes áreas de atuação.

A conclusão de cursos de pós-graduação foi referida por $52,2 \%$ dos nutricionistas participantes do trabalho (Tabela 1), percentual próximo do retrato ampliado do nutricionista no Brasil realizado pelo $\mathrm{CFN}^{10}$, em que $55,9 \%$ dos entrevistados relataram conclusão de curso de pós-graduação.

Gambardella et al. ${ }^{13}$, investigando a situação profissional de nutricionistas em São Paulo, observaram que $37,1 \%$ dos participantes realizavam algum aprimoramento, especialização ou pós-graduação. Ao descrever aspectos relacionados à atuação profissional de egressos da Universidade Federal de Santa Catarina, Alves et al. ${ }^{14}$ observaram que $61,8 \%$ dos nutricionistas cursaram ou estavam cursando algum tipo de pós-graduação. Os baixos recursos financeiros e a escassez de tempo estavam entre os principais motivos alegados pelos nutricionistas que não cursaram pós-graduação, identificados por Rodrigues et al. ${ }^{12}$. No presente estudo, a grande participação de profissionais recém-formados pode explicar parcialmente o elevado percentual de nutricionistas sem pós-graduação $(47,8 \%)$. 
Dentre os nutricionistas que participaram deste estudo, 68,4\% referiram alguma experiência profissional antes de atuarem no PNAE. Alimentação coletiva, nutrição clínica e saúde pública foram as áreas de atuação profissional mais mencionadas. Segundo Rodrigues et al. ${ }^{12}$, a partir da avaliação de relatos de nutricionistas egressos da Escola de Nutrição da Universidade Federal de Ouro Preto/MG, o primeiro local de atuação profissional, na maioria dos casos observados, foi uma Unidade de Alimentação e Nutrição (UAN). Contudo, devido às condições de trabalho desfavoráveis e ao surgimento de outras oportunidades, esses profissionais migravam para outras áreas de atuação, embora tenha sido identificado que aquela era a área com melhor remuneração, situando-se na média de 5,34 salários-mínimos.

Em estudo conduzido na cidade de São Paulo, Gambardella et al. ${ }^{13}$ apresentaram resultados mais promissores do que os exibidos neste trabalho, uma vez que $59,1 \%$ dos nutricionistas atuantes nas mais diversas áreas da nutrição auferiam rendimentos superiores a dez salários-mínimos, enquanto Prado \& Abreu ${ }^{15}$, em estudo realizado no Rio de Janeiro mostraram renda média de 7,5 salários-mínimos. Vale ressaltar que, apesar da ausência de estudos que identifiquem o perfil do profissional atuante no PNAE, dificultando a análise comparativa dos resultados desse trabalho, é possível inferir que o grupo de profissionais participantes da presente pesquisa encontra-se em situação desfavorável do ponto de vista financeiro, comparado com a remuneração de nutricionistas atuantes nas diversas áreas em outras regiões do País ${ }^{11}$.

Os obstáculos enfrentados pelos nutricionistas na criação das especialidades, bem como as dificuldades em demarcar os objetivos da profissão são reflexos da baixa capacidade de decisão, da desmobilização da categoria e da falta de valorização atribuída à classe ${ }^{11}$, embora algumas conquistas mereçam destaque, como a ampliação dos campos de atuação profissional e o ascendente processo de especialização ${ }^{16}$. Assim, deve-se ponderar que a busca por autonomia per- corre o desejo de obter melhores salários, o que, por sua vez, pode implicar acúmulo de atividades e responsabilidades. No âmbito do programa de alimentação escolar, a assunção de atribuições, além daquelas previstas em lei, pode gerar incompatibilidades com o bom desenvolvimento das ações de nutrição, dessa maneira limitando os resultados visados pelas diretrizes do PNAE.

Embora esteja em vigor a Resolução do CFN no 465/2010 ${ }^{17}$, que dispõe sobre as atribuições do nutricionista no Programa de Alimentação Escolar (PAE), este estudo teve como referência a Resolução do CFN nº 358/2005, vigente à época de sua realização. Esta considerava, além das atividades técnicas já apresentadas nas Tabelas 2 e 3, outras ações de acordo com a necessidade, complexidade do serviço ou estrutura operacional do $\mathrm{PAE}^{7}$.

Adicionalmente, a Lei $n^{\circ} 11.947$, nos artigos $11^{\circ}$ e $12^{\circ}$, amplia as ações desse profissional, recomendando que o planejamento de cardápios considere a cultura e tradições alimentares locais, de modo sustentável e com base na diversificação agrícola regional. Além disso, contempla a educação alimentar e nutricional nas escolas, considerando como estratégias, entre outras, a oferta da alimentação saudável, a implantação e manutenção de hortas escolares pedagógicas, a inserção do tema alimentação saudável no currículo escolar, a realização de oficinas culinárias experimentais com os alunos, a formação da comunidade escolar, bem como o desenvolvimento de tecnologias sociais que a beneficiem, com vistas à preservação e recuperação da cultura alimen$\operatorname{tar}^{1,2}$. Alguns desses aspectos já representam uma realidade em determinadas localidades do país, revelando um quadro bastante favorável de melhora qualitativa da alimentação escolar ${ }^{18-20}$. Nesse sentido, tem sido constatado que a prática da alimentação saudável e a modificação de hábitos alimentares requerem atividades educativas que visem à autonomia dos sujeitos beneficiários das ações no processo de escolha dos alimentos, considerando os diversos fatores que determinam o comportamento alimentar $r^{3,21,22}$. 
Vale salientar que, de acordo com a Resolução do CFN n 465/2010 17, as ações de educação alimentar e nutricional do escolar são uma atividade obrigatória do nutricionista, considerando-se ainda o seu papel estratégico na promoção da alimentação saudável na escola. No entanto, apenas 33,18\% dos nutricionistas entrevistados no presente estudo revelaram realizar frequentemente essa atividade. Muito embora o CFN ${ }^{10}$ tenha apontado em seu estudo que todos os setores realizam com frequência alguma forma de educação alimentar em seu trabalho, é ainda incipiente o uso de material técnico-profissional produzido pelos órgãos do Ministério da Saúde, indicando uma desvinculação ou desconhecimento dos instrumentos de políticas públicas para a área. Dessa maneira, conhecer os instrumentos que normatizam a execução do Programa e dispõem sobre as atribuições do profissional, constitui etapa essencial e primordial para o trabalho do nutricionista que atua na alimentação escolar.

Nesse sentido, cursos de formação continuada despontam como importantes alternativas na promoção da troca de experiências, atualização de informações, valorização e motivação da prática profissional, uma vez que, sem reflexão e avaliação da sua práxis, o bom funcionamento do Programa não é possível, a exemplo da recente experiência de formação desenvolvida para merendeiras de escolas estaduais de Salvador (BA ${ }^{23}$. Outro aspecto relevante é a integração do trabalho entre os atores envolvidos no PNAE: conselheiros, merendeiros, gestores escolares, professores, gestores municipais, coordenadores pedagógicos e coordenadores da alimentação escolar. O estabelecimento das parcerias entre os atores, através de um trabalho integrado é de fundamental importância para o alcance dos objetivos do Programa, para sua melhoria e para a otimização, reconhecimento e valorização das ações.

Nos últimos cinco anos, o FNDE/MEC tem realizado investimentos nos cursos de formação continuada para os atores do PNAE, por meio de parceria com os Centros Colaboradores em Ali- mentação e Nutrição do Escolar, visando à qualificação dos profissionais e à sua atualização em novos temas, como, por exemplo, a aquisição de gêneros alimentícios da agricultura familiar, garantida pela Resolução/CD/FNDE $n^{\circ} 38$, de 16 de julho de 2009'.

Contudo, deve-se ponderar que as atribuições do nutricionista do PNAE devem ser analisadas frente aos parâmetros numéricos estabelecidos pela Resolução do CFN n ${ }^{\circ} 465 / 2010^{17}$, a qual determina a proporcionalidade entre o número de alunos e o de nutricionista, bem como a carga horária técnica mínima semanal recomendada. Em estudo realizado entre 2003 e 2004 sobre a Alimentação Escolar no Brasil, observou-se que em torno de $37 \%$ dos estados brasileiros apresentaram graves problemas de dimensionamento de recursos humanos (Espírito Santo, Mato Grosso do Sul, Paraíba, Paraná, Piauí, Rio Grande do Norte, Rio Grande do Sul, Roraima, Santa Catarina e Tocantins), enquanto apenas $25,9 \%$ possuíam equipes mais completas e em número compatível com as atividades desenvolvidas (Acre, Amazonas, Distrito Federal, Goiás, Maranhão, São Paulo e Sergipe ${ }^{24}$. Dessa forma, frente aos objetivos do Programa e à abrangência das atividades propostas, deve-se considerar o reduzido quadro de pessoal, que inviabiliza a realização plena das ações previstas.

Uma das responsabilidades do nutricionista vinculado ao setor de alimentação escolar é a elaboração de cardápios. Estes devem ser planejados com a finalidade de suprir, no mínimo, 30\% das necessidades nutricionais diárias dos alunos de escolas localizadas em comunidades indígenas e remanescentes de quilombolas, e $20 \%$ das necessidades de alunos matriculados na educação básica em período parcial. Além da atenção às necessidades nutricionais, a construção dos cardápios deve ser pautada na promoção de hábitos alimentares saudáveis, no respeito aos hábitos e cultura alimentar, e na vocação agrícola de cada localidade ${ }^{25}$. O planejamento adequado do cardápio e sua efetiva execução, além da atenção ao aporte nutricional mínimo, não só atende aos 
objetivos do Programa, como também pode evitar problemas relacionados às irregularidades no fornecimento dos gêneros ${ }^{26,27}$.

No modelo de gestão descentralizada do PNAE, a infraestrutura necessária para a preparação das refeições é de responsabilidade dos municípios, o que constitui dificuldade para muitos gestores ${ }^{26,28,29}$. Por outro lado, essa modalidade de gestão tem facilitado a adequação dos cardápios aos hábitos alimentares locais, garantindo a introdução de alimentos in natura no cardápio escolar ${ }^{26}$, corroborando as ações de educação alimentar e nutricional, sanitária e ambiental, conforme prevê a Lei n 11.947 , em seu artigo $17^{\circ 1}$.

Os resultados encontrados neste estudo mostraram que as atividades que integram as ações do nutricionista no âmbito do PNAE (apresentadas nas Tabelas 2 e 3) foram significativamente associadas com o seu tempo de atuação no Programa. Quando observado o grupo de atividades, nota-se que os nutricionistas com tempo de atuação entre dois e cinco anos realizavam frequentemente as ações. Por outro lado, nutricionistas com tempo de PNAE superior a cinco anos realizavam as ações com frequência inferior aos demais grupos. Conforme identificado por Analoni ${ }^{30}$, ao analisar a situação de trabalho, a inserção no mercado e a posição do nutricionista em empresas de alimentação coletiva em Minas Gerais, esse profissional passou a exercer funções predominantemente administrativas, assumindo um perfil de profissional organizacional, gerente ou supervisor, em decorrência do desgaste, da baixa valorização e da insatisfação com o nível de utilização dos seus conhecimentos técnicos.

Para o grupo de nutricionistas estudado nesta abordagem, embora haja um alto nível de satisfação com a atuação profissional, ocorre grande descontentamento com as condições de trabalho (Figura 1). Assim, considerando a importância de cada atividade para o bom desempenho do Programa, torna-se necessário criar condições favoráveis de trabalho, rever os quadros de pes- soal, promover o trabalho integrado dos atores do PNAE e, sobretudo, favorecer a autonomia e a criatividade do nutricionista ${ }^{28,31}$, na tentativa de agregar prazer e satisfação à atividade desenvolvida.

\section{O N CLUS Ã O}

Considerando os resultados obtidos no presente estudo, pode-se deduzir que, no âmbito do PNAE, o nutricionista assume responsabilidades que fazem interface com outras áreas do conhecimento, destacadamente com a Educação e a Administração. Paralelamente, apesar da satisfação com a atuação profissional, os participantes sentiam-se pouco satisfeitos em relação às condições de trabalho. No enfrentamento dessas condições, o profissional encontrou dificuldades, a começar pelo tipo de vínculo empregatício estabelecido com o Programa, o que muitas vezes exigiu a assunção de outras atividades profissionais na área da Nutrição, ou mesmo a responsabilidade técnica por outros municípios. Agregou-se a esse fato a baixa remuneração auferida no PNAE, que impacta negativamente na satisfação profissional e na dedicação dispensada ao Programa.

É importante destacar que a formação continuada dos nutricionistas para atuar no referido campo deve ser priorizada, uma vez que os cursos de graduação não têm acompanhado o crescimento do trabalho na área. Ou seja, ainda há pouco investimento acadêmico nesse campo de atuação profissional, com incipientes cursos e componentes curriculares.

Deve-se ainda aqui considerar que o processo de consolidação da atuação do nutricionista no campo da alimentação escolar ao longo das últimas décadas também poderá ser responsável por um processo de interiorização da profissão, frente à obrigatoriedade de contratação desse profissional nos municípios, como responsável técnico do Programa. Dessa forma, a modalidade de educação continuada, a exemplo dos cursos à distância, pode constituir estratégia para a constante qualificação profissional. 
São necessários mais estudos sobre o tema, objetivando investigar a atuação do nutricionista na área da alimentação escolar. Dessa forma, espera-se que este trabalho possa colaborar para a implementação de estratégias que contribuam para a melhoria das condições de trabalho do nutricionista no PNAE, na perspectiva da promoção da saúde no contexto escolar.

\section{A GRADECIMENTO}

Ao Fundo Nacional de Desenvolvimento da Educação, do Ministério da Educação, Brasil, que possibilitou a realização deste trabalho, mediante disponibilidade de recursos humanos e financeiros.

\section{REFER Ê N CIAS}

1. Brasil. Lei $n^{\circ} 11.947$, de 16 de junho de 2009. Dispõe sobre o atendimento da alimentação escolar e do programa dinheiro direto na escola aos alunos da educação básica; altera as Leis n 10 880, de 9 de junho de 2004, 11 273, de 6 de fevereiro de 2006, 11 507, de 20 de julho de 2007; revoga dispositivos da Medida Provisória n².178-36, de 24 de agosto de 2001 e a Lei $n^{\circ} 8$ 913, de 12 de julho de 1994 e dá outras providências. Diário Oficial da União. 200916 jun; p.2, Seção 1.

2. Brasil. Ministério da Educação. Fundo Nacional de Desenvolvimento da Educação [Internet]. Resolução/FNDE/CD/n 38, de 16 de julho de 2009. Brasília: Ministério da Educação; 2009 [acesso 2010 jan 10]. Disponível em: <http://www.fnde.gov.br/ index.php/legis-resolucoes>.

3. Caniné ES, Ribeiro VMB. A prática do nutricionista em escolas municipais do Rio de Janeiro: um espaço-tempo educativo. Ciên Educ. 2007; 13(1): 47-70. doi: 10.1590/S1516-73132007000100004.

4. Costa EQ, Ribeiro VMB, Ribeiro ECO. Programa de alimentação escolar: espaço de aprendizagem e produção de conhecimento. Rev Nutr. 2001; 14(3): 225-9. doi: 10.1590/S1415-52732001000300009.

5. Pipitone MAP. A relação saúde e educação na escola de $1^{\circ} \mathrm{Grau}$. Aliment Nutr.1995; 15(65):48-52.

6. Zancul MS, Oliveira JED. Considerações sobre ações atuais de educação alimentar e nutricional. Aliment Nutr. 2007; 18(2):223-7.

7. Conselho Federal de Nutricionistas. Resolução CFN $n^{\circ} 358 / 2005$. Dispõe sobre as atribuições do Nutricionista no âmbito do Programa de Alimentação
Escolar (PAE) e dá outras providências. Brasília; 2005 [acesso 2009 set 14]. Disponível em: <http://ftp. fnde.gov.br/web/resolucoes_2005/res358_ 2005_cfn.pdf>.

8. Santos LAS, Silva MCM, Santos JM, Assunção MP, Portela ML, Soares MD, et al. Projeto pedagógico do programa de graduação em nutrição da Escola de nutrição da Universidade Federal da Bahia: uma proposta em construção. Rev Nutr. 2005; 18(1): 105-17. doi: 10.1590/S1415-5273200500010 0010.

9. Brasil. Ministério da Educação. Fundo Nacional de Desenvolvimento da Educação. Resolução/FNDE/ $\mathrm{CD} / \mathrm{n}^{\circ} 32$, de 10 de agosto de 2006. Brasília: Ministério da Educação; 2006 [acesso 2009 set 14]. Disponível em: <http://ftp.fnde.gov.br>.

10. Conselho Federal de Nutricionistas. Inserção profissional dos nutricionistas no Brasil. Brasília: CFN; 2006 [acesso 2011 ago 29]. Disponível em: <http:// www.cfn.org.br/novosite/pdf/pesquisa.pdf>.

11. Akutsu RC. Brazilian dieticians: professional and demographic profiles. Rev Nutr. 2008; 21(1):7-19. doi: 10.1590/S1415-52732008000100002.

12. Rodrigues KM, Peres F, Waissmann W. Condições de trabalho e perfil profissional dos nutricionistas egressos da Universidade Federal de Ouro Preto, Minas Gerais, entre 1994 e 2001. Ciênc Saúde Coletiva. 2007; 12 (4):1021-31. doi: 10.1590/S1413-8 1232007000400023.

13. Gambardella AMD, Ferreira CF, Frutuoso MFP. Situação profissional de egressos de um curso de nutrição. Rev Nutr. 2000; 13(1):37-40. doi: 10.15 90/S1415-52732000000100005.

14. Alves E, Rossi CE, Vasconcelos FAG. Nutricionistas egressos da Universidade Federal de Santa Catarina: áreas de atuação, distribuição geográfica, índices de pós-graduação e de filiação aos órgãos de classe. Rev Nutr. 2003; 16(3):295-304. doi: 10.15 90/\$1415-52732003000300007.

15. Prado SD, Abreu MSD. Nutricionista: onde trabalha? quais suas condições de trabalho? Rev Nutr. 1991; 4(1/2):65-92.

16. Vasconcelos FAG. O nutricionista no Brasil: uma análise histórica. Rev Nutr. 2002; 15(2):127-38. doi: 10.1590/S1415-52732002000200001.

17. Conselho Federal de Nutricionistas. Resolução CFN $n^{\circ} 465 / 2010$. Dispõe sobre as atribuições no Nutricionista, estabelece parâmetros numéricos mínimos de referência no âmbito do Programa de Alimentação Escolar e dá outras providências. Brasília: CFN; 2001 [acesso 2011 set 9]. Disponível em: <http://www.fnde.gov.br/index.php/ae-legislacao>.

18. Vianna RPT, Tereso MJA. O programa de merenda escolar de Campinas: análise do alcance e limita- 
ções do abastecimento regional. Rev Nutr. 2000; 13(1):41-9. doi: 10.1590/S1415-5273200000010 0006.

19. Muniz VM, Carvalho AT. O Programa de Alimentação Escolar em município do estado da Paraíba: um estudo sob o olhar dos beneficiários do programa. Rev Nutr. 2007; 20(3):285-96. doi: 10.1590/S1 415-52732007000300007.

20. Chaves LG, Mendes PNR, Brito RR, Botelho RBA. O programa nacional de alimentação escolar como promotor de hábitos alimentares regionais. Rev Nutr. 2009; 22(6):857-66. doi: 10.1590/S1415-5 2732009000600007.

21. Neutzling MB, Assunção MCF, Malcon MC, Hallal PC, Menezes AMB. Hábitos alimentares de escolares adolescentes de Pelotas, Brasil. Rev Nutr. 2010; 23(3):379-88. doi: 10.1590/S1415-527320100003 00006.

22. Dalla Costa MC, Cordoni Junior L, Matsuo T. Hábito alimentar de escolares adolescentes de um município do oeste do Paraná. Rev Nutr. 2007; 20(5): 461-71. doi: 10.1590/S1415-527320070005000 02.

23. Leite LG, Cardoso RCV, Góes JAW, Figueiredo KVNA, Silva EO, Bezerril MM, et al. Formação para merendeiras: uma proposta metodológica aplicada em escolas estaduais atendidas pelo programa nacional de alimentação escolar, em Salvador, Bahia. Rev Nutr. 2011; 24(2):275-85. doi: 10.1590/ S1415-52732011000200008.

24. Stolarski MC. Caminhos da alimentação escolar no Brasil: análise de uma política pública no período de 2003-2004 [dissertação]. Curitiba: Universidade Federal do Paraná; 2005.

25. Brasil. Ministério da Educação. Fundo Nacional de Desenvolvimento da Educação. Alimentação escolar: dados estatísticos. Brasília: Ministério da
Educação; 2009 [acesso 2009 set 04]. Disponível em: <http://www.fnde.gov.br/index.php/aelegislacao>.

26. Spinelli MAS, Canesqui AM. Descentralização do Programa de Alimentação Escolar em Cuiabá: 1993-1996. Rev Nutr. 2004; 17(2):151-65. doi: 10.1 590/S1415-52732004000200002.

27. Spinelli MAS, Canesqui AM. O programa de alimentação escolar no estado de Mato Grosso: da centralização à descentralização (1979-1995). Rev Nutr. 2002; 15(1):105-17. doi: 10.1590/\$1415-5 2732002000100011.

28. Santos LMP, Araújo MPN, Martins MC. Avaliação de políticas públicas de segurança alimentar e combate à fome no período 1995-2002. 4: Programa Nacional de Alimentação Escolar. Cad Saúde Pública. 2007; 23(8):2681-93. doi: 10.1590/S0102-3 $11 \times 2007001100016$.

29. Pipitone MAP, Ometto AMH, Silva MV, Sturion GL, Furtuoso COM, Oetterer M. Atuação dos conselhos municipais de alimentação escolar na gestão do programa nacional de alimentação escolar. Rev Nutr. 2003; 16(2):143-54. doi: 10.1590/S1415-52 732003000200001.

30. Analoni JA. Situação de trabalho dos nutricionistas em empresas de refeições coletivas de Minas Gerais: trabalho técnico, supervisão ou gerência? Rev Nutr. 1999; 12(3):241-60. doi: 10.1590/S1415-527319 99000300005.

31. Bosi MLM. Trabalho e subjetividade: cargas e sofrimento na prática da nutrição social. Rev Nutr. 2000; 13(2):107-15. doi: 10.1590/\$1415-5273200000 0200005.

Recebido em: 25/1/2011

Versão final reapresentada em: 21/11/2011

Aprovado em: 9/1/2012 